\title{
Graft Protection Against Cold Ischemia Preservation: An Institute George Lopez 1 and Histidine- tryptophan-ketoglutarate Solution Appraisal
}

\author{
Arnau Panisello-Rosello ${ }^{1^{*}}$, Carlos Castro-Benítez ${ }^{2 *}$, Alexandre Lopez $^{2}$, Sravan Balloji ${ }^{1}$, Emma- \\ Folch $^{1}$, René Adam ${ }^{2}$, Joan Roselló-Catafau ${ }^{1}$ \\ 1 Institut d'Investigacions Biomèdiques de Barcelona (IIBB), CSIC-IDIBAPS, Catalonia, Barcelona, \\ Spain; 2 Centre Hepato-Biliare, AP-P-HP Hôpital Paul Brousse, Inserm U776, Univesité Paris-Sud, \\ Villejuif, 75008-Paris, France; Experimental Hepatic Ischemia-Reperfusion Unit,
}

Author Contribution: Panisello A and Castro-Benítez C, both contributed equally to this work. Panisello A and Castro- Benítez C. provided protocols and analyzed data, established the animal experimental model used in this study and carried out the experimental work. Lopez A and Balloji S were carried out analyses Folch- Puy E, Adam R and Roselló-Catafau J designed the study, coordinate the experiments and wrote the paper

Supported by:Instituto de Salud Carlos III (ISCIII) through the FIS project PI12/0056 cofunded by FEDER from Regional Development European Funds (European Union). Sravan Bellogi was fellowshipholder from project FOIE GRAS, from the European Union's Horizon 2020 Research and Innovation programme under the Marie Sklodowska-Curie Grant Agreement No. 722619

Corresponding author: Dr. Joan Roselló-Catafau, Experimental Hepatic Ischemia-Reperfusion Unit, IIBB-CSIC, C/ Rosselló 161, 7th floor, E-08036-Barcelona, Spain. jrcbam@iibb.csic.es 
Telephone: +34-933638333 Fax: +34-933638301

Presented at the $15^{\text {th }}$ Congress of the Brazilian Transplantation Society (ABTO-2017) and the $16^{\text {th }}$ LusoBrazilian Congress on Transplantation to be held October 18-21, 2017 in Foz do Iguaçu, Brazil.

Key Words:

cold ischemia-reperfusion injury, liver graft washing out, IGL-1 preservation solution, HTK preservation solution

\section{ABSTRACT}

Cold storage of organs in preservation solutions, such as Institute George Lopez 1 (IGL-1) or histidinetryptophan-ketoglutarate (HTK), is a mandatory step for organ transplantation. This preservation leads to an ischemic injury that affects the outcome of the organ. This article studies the liver graft eluate after organ recovery using IGL-1 or HTK solutions. We explore the influence of the volume used for washing out the liver and the consequences in the graft preservation when both solutions are used. Livers were washed out with different volumes of HTK and IGL-1 according to manufacturers' instructions and then preserved in both solutions for 24 hours at $4 \mathrm{C}$. Tissue and eluates were collected for subsequent analyses. We measured transaminases (aspartate aminotransferase and alanine aminotransferase), histology by hematoxylin/eosin staining, and red blood cell and hemoglobin counts, respectively. After washing out and cold storage, the IGL-1 processed livers showed better preservation than those with HTK solution; however, in this latter case, an important accumulation of erythrocytes was found when compared to IGL-1. These data were consistent with the higher hemoglobin and red blood cell counts observed for IGL-1 eluates after 24 hours. The volume used for washing out the organ depends on the 
composition and properties of the organ preservation solutions (ie, IGL-1 and HTK); this is an important factor for the graft cold preservation. The total volume used for washing out the graft should be considered because it has a direct impact on the total cost for clinical transplantations.

\section{INTRODUCTION}

COLD ischemia reperfusion injury is a limiting step for the success of liver transplantation. This is injury can be modulated during graft cold storage by the use of different organ preservation solutions, including Institute George Lopez 1 (IGl-1) and histidine-tryptophanketoglutarate (HTK) which are currently used for abdominal transplantation purposes (Table 1). Recently it has been reported by the European Liver Transplant Registry (ELTR) that HTK shows some limitations in front of other solutions, including IGL-1 solution [1]. ELTR results were in accordance with those previously published by the United Network for Organ Sharing (UNOS) [2]. Taking this into account, we have explored the relevance of pathophysiological mechanisms involved in graft preservation when IGL-1 and HTK solutions were used [3]. We studied an aspect that has been poorly investigated; that is, the influence of the volumes used for washing out and preserving organs with different solutions such as IGL-1 and HTK (used according to the manufacturer instructions) [4e6] on the quality of graft preservation. Here we present a detailed washing out appraisal for comparing IGL-1 and HTK solutions in a controlled experimental preservation rat model when a longer time of cold preservation time (24 hours at 4 C) was used. Results show that the volumes of the organ preservation solutions should be considered during graft preservation.

\section{MATERIALS AND MEHTODS}

\section{Animals:}

Sprague-Dawley rats weighing $250 \mathrm{~g}$ from Charles River (France) were used. All animals had free access to water and dry food. All animals were used in accordance with protocols approved on July 14, 2016 (NO-483116). The study was performed in accordance with the European Union Directive 2010/63/EU for animal experiments and approved by the Ethics Committees for Animal Experimentation of the 
University of Barcelona (Directive 396/12). Animals were randomly distributed into groups as described below.

\section{Experimental Design:}

In a sterile room, we anesthetized the rats (Sprague-Dawley, male) with isoflurane with a silicone mask at $4 \%$ during 1 to 2 minutes for induction, and then $2 \%, 2$ 1/min air flow for liver procurement. After cleaning and shaving the abdomen, we performed a medium xifopubian incision. Retractors of the abdominal wall were placed and then the small intestine was moved and covered with wet gauze. Sections of the falciform, left triangular, and gastrohepatic ligaments were done before the division of the left diaphragmatic vein from the suprahepatic IVC. Next, the hepatoesophageal ligament was cut and the artery coagulated. To isolate the infrahepatic IVC down to the left right renal vein, we sutured using 4-0 silk that had been placed around it. We proceeded to separate the proper hepatic artery and dissect the portal vein and the bile duct to fix the hepatic hilum. Then, 2 IU of heparin was injected through the dorsal vein of the penis and we used a bulldog clamp for the abdominal aorta and the IVC just before its ileac bifurcation and diaphragm transection. A bulldog clamp was placed at the suprahepatic IVC level just after the heart. A cannula was placed in the aorta and a $14 \mathrm{G}$ catheter was placed in the infrahepatic IVC and secured with 4-0 silk sutures. We flushed livers with the $40 \mathrm{~mL}$ of IGL-1 or $100 \mathrm{~mL}$ of HTK through the aorta and we collected effluents drained by the infrahepatic IVC through the catheter (t0 at the beginning of the flush, $\mathrm{t} 1$ after $40 \mathrm{~mL}$ of both solutions, and $\mathrm{t} 2$ just in the HTK group after $100 \mathrm{~mL}$ ). We also flushed with $10 \mathrm{~mL}$ of IGL-1 or $25 \mathrm{~mL}$ of HTK through a $22 \mathrm{G}$ catheter by the portal vein and perfusate was collected ( $\mathrm{t}$ 1/4 Portal). Finally, we cut the suprahepatic and infrahepatic IVCs, portal vein, and bile duct just below the catheter, and liver specimens were placed into a basin filled with the preservation solutions IGL1 or HTK, respectively, for 24 hours at 4 C (Fig 1). After 24 hours, all livers were rinsed with $20 \mathrm{~mL}$ Ringer lactate solution and samples were obtained from the flush ( $\mathrm{t} 1 / 424$ hours). Liver samples were then stored at $80 \mathrm{C}$ for the subsequent biochemical determinations. 
Experimental groups:

- Group 1 (HTK; $n=6$ ): After organ wash and recovery, the livers were stored in HTK solution for 24 h at $4{ }^{\circ} \mathrm{C}$ as previously described

- Group 2 (IGL-1; $n=6$ ): After organ wash and recovery, the livers were stored in IGL-1 preservation solution for $24 \mathrm{~h}$ at $4{ }^{\circ} \mathrm{C}$ as previously described

\section{Determinations:}

Liver injury : Hepatic injury was evaluated by measuring alanine aminotransferase (ALT) and aspartate aminotransferase (AST) levels using commercial kits from RAL (Barcelona, Spain), as previously reported [3]. Briefly, $100 \mathrm{~mL}$ of effluent washout liquid or perfusate was added to $1 \mathrm{~mL}$ of the substrate provided by the commercial kit, and then transaminase activity was measured at $340 \mathrm{~nm}$ with an ultraviolet spectrometer and calculated following the supplier's instructions.

Red Blood Cell count: Red blood cells (RBCs) were counted using a Neubauer chamber under a microscope (original magnification 10) using standardized protocol. All rats were analyzed at time 0 and after portal and 24 hours after washing. Hemoglobin. Hemoglobin (Hb) levels were analyzed using Drabkin's method with human hemoglobin from Sigma-Aldrich Company (Madrid, Spain) as standard. All rats were analyzed at time 0 and after portal and 24 hours after washing.Hemoglobin $(\mathrm{Hb})$ : Hemoglobin levels were analyzed using Drabkins method using Human hemoglobin from Sigma-Aldrich Company (Madrid, Spain) as standard. All rats were analyzed at time 0 and after portal and $24 \mathrm{~h}$ washing.

\section{Histology:}

Histologic cuts of livers after 24-hour preservation were mounted and prepared for hematoxylin-eosin staining. Ten random fields were selected in each sample of the microscope and erythrocytes were 
counted at original magnification 40 . The sum of numbers of 10 fields is the total number of cells in the samples.

\section{Statistics:}

Data are expressed as mean standard error, and were compared statistically by variance analysis, followed by the Mann-Whitney U test using GraphPad Prism version 4.02 for Windows (GraphPad Prism software; accessed: May 17, 2004). P <.05 was considered significant.

\section{RESULTS}

\section{Quantification of hemoglobin in perfusate samples:}

Cleanness of the perfusate after liver wash was determined from levels of RBCs and Hb (Fig 2). Data show significant differences in both of the parameters analyzed. In RBC counts, there is no difference at time 0 of the washing between HTK and IGL-1, although we can find significantly more erythrocytes in the perfusate after using IGL-1 solution compared with HTK after portal and 24-hour washings (Fig 2A). $\mathrm{Hb}$ analysis of the perfusate showed significant differences in the last washing, after the 24-hour preservation (Fig 2B). Cleanness of the perfusate after liver wash was measured as red blood cells (RBCs) and hemoglobin $(\mathrm{Hb})$. Data shows significant differences in both of the parameters analyzed. In RBCs counting there is no difference at time zero of the washing between HTK and IGL-1, although we can find significantly more erythrocytes in the perfusate after using IGL-1 solution compared with HTK after portal and $24 \mathrm{~h}$ washings. Hemoglobin analysis of the perfusate showed significant differences in the last washing, after the $24 \mathrm{~h}$ preservation.

\section{Erythrocytes quantification in tissue samples at the end of preservation period:}

After 24 hours, histologic samples were prepared and stained with hematoxylin-eosin, and RBCs were counted in 10 random fields for each of them (Fig 3A). As Figure 3B shows, there are significantly more erythrocytes in the livers preserved with HTK than in those preserved with IGL-1. 


\section{Liver injury:}

Liver injury, measured using ALT and AST levels, showed significant lower levels in livers treatedwith IGL-1 solution (Fig 4).

\section{DISCUSSION}

The results presented show how when using the washing-out and preservation volume recommendations of manufacturers from HTK and IGL (which means using 2.5 times more volume of HKT than IGL-1) the quality of rinsing as measured by the quantification of erythrocytes in liver tissue and perfusate after the whole period of preservation is higher in HTK when compared with IGL-1. This correlated with the higher measured liver damage in HTK as reported by others [3,7e9]. Recommendation for HTK solution call for the use of approximately $300 \mathrm{~mL} /$ body weight. On the other hand, other information suggests that between $10 \mathrm{~L}$ and $8 \mathrm{~L}$ should be used for the liver [5]. For this reason we chose to infuse in the experimental model using the maximum recommendation (reduced to the scale of the rat liver preservation model) assuming that with this amount of solution the most efficient washing-out and preservation efficiency would be obtained. Therefore, the volume of HTK is approximately 2.5 times higher than IGL-1 (Fig 1).

The higher volume recommended for HTK is based on the fact that this solution has a very low viscosity; therefore, when compared to University of Wisconsin (UW) solution, it flows 3 times faster than UW [4]. As a consequence, it makes sense to think that the volume recommended for HTK is almost 3 times higher than UW. As for IGL-1, the same volume as UW is recommended, which also follows for other solutions such as Celsior [6].

Furthermore, the capacity of washing-out and preservation of a given solution is partially related to the presence of oncotic agent. The lack of oncotic agent, such as the case of HTK, produces a certain degree of interstitial edema that collapse small sinusoids [10]. On the contrary, in IGL-1 the oncotic agent 
polyethylene glycol (PEG) $35 \mathrm{KD}$ (PEG 35) limits interstitial edema and so favors flushing of even small vessels. This has been shown especially in steatotic livers experimentally [11]. Therefore, the positive effect of PEG in IGL-1 is present but with the additional property of still providing a low viscosity to the solution when compared to UW, and similar to HTK. Furthermore, PEG 35 does not promote RBC aggregation in contrast to hydroxyethyl starch present in UW, facilitating the wash-out of the graft even through the small sinusoids [12].

Finally, the volumes used for washing-out and preservation have a direct financial implication because the total cost of preservation is not only related to the cost per liter of solution but also with the combination with the total volume of solution used.

\section{REFERENCES}

1- Adam R, Delvart V, Karam V et al. Compared efficacy of preservation solutions in liver transplantation: a long-term graft outcome study from the European Liver Transplant Registry. Am J Transplant. 2015 Feb;15(2):395-406. doi: 10.1111/ajt.13060.

2- Stewart ZA, Cameron AM, Singer AL et al. Histidine-tryptophan-ketoglutarate (HTK) is associated with reduced graft survival in deceased donor livers, especially those donated after cardiac death. Am $\mathbf{J}$ Transplant 2009; 9: 286-293.

3- Arnau Panisello-Roselló, Eva Verde, Mohamed Amine Zaouali et al. The Relevance of the UPS in Fatty Liver Graft Preservation: A New Approach for IGL-1 and HTK Solutions Int. J. Mol. Sci. In press.

4- Custodiol HTK Solution PI available at http://www.custodiol.com/prescribing-info/ 
5- Custodiol HTK Solution Abdominal Brochure at http://www.custodiol.com/hansjb/wpcontent/uploads/2016/02/DigitalAbdominalBooklet2.9.16.pdf

6- Products information at http://www.groupe-igl.com

7- Viederkehr et al. Use of IGL-1 preservation solutionin liver transplantation. Transplant Proc. 2014, 461809-11.

8- M.H. Meine et al. Comparison between IGL-1 and HTK preservation solutions in deceased donor liver transplantation.. Transplant Proc. 2015, 47: 888-93.

9- Vincent Karam et al. Impact of Preservation Solution On Early Allograft Dysfunction (EAD) and Graft Survival after Liver Transplantation (LT) Using Expanded-criteria-Donors (ECD) .The 2017 Joint International Congress of ILTS, ELITA \& LICAGE May 24 - 27, 2017, Prague, Czech Republic.

10- Bizugas M, Jablonski P, Thomas AC et al. Evaluation of UW solution in a rat kidney preservation model. I. Effect of hydroxyethyl starch and electrolyte composition. Transplantation 1990;49:872.

11- Ben Mosbah I, Roselló-Catafau J, Franco-Gou R et al. Preservation of steatotic livers in IGL-1 solution. Liver Transpl. 2006; 12:1215.

12- Mosbah IB, Franco-Gou R, Abdennebi HB, et al. Effects of polyethylene glycol and hydroxyethyl starch in University of Wisconsin preservation solution on human red blood cell aggregation and viscosity. Transplant Proc. 2006 Jun;38(5):1229-35. 


\begin{tabular}{|lccccc|}
\hline & IGL-1 & HTK & & IGL-1 & H TK \\
\hline Colloids (g/1) & & & Electrolytes (mmol/1) & & \\
Polyethylene glycol-35 & 1 & & $\mathrm{~K}+$ & 25 & 9 \\
Antioxydants (mmol/1) & & & $\mathrm{Na}+$ & 125 & 15 \\
Glutathione & 3 & & $\mathrm{Mg} 2+$ & 5 & 4 \\
Allopurinol & 1 & & $\mathrm{Cl}-$ & 50 \\
Precursors (mmol/1) & & & SO4 2- & 5 & \\
Adenosine & 5 & & Impermeants (mmol/1) & \\
Ketoglutarate & & 1 & Rafffinose & 30 & \\
pH & 7,4 & 7,2 & Lactobionic acid & 100 & \\
Osmolarity (mosmol/1) & 290 & 310 & Mannitol & & 30 \\
Buffers (mmol/1) & & & & & \\
Diphosphate & 25 & & & & \\
Histidine & & 180 & & \\
Histidine-HC1 & & 18 & & \\
Tryptophan & & 2 & & \\
\hline
\end{tabular}

Table 1: Composition of HTK and IGL-1 solutions.

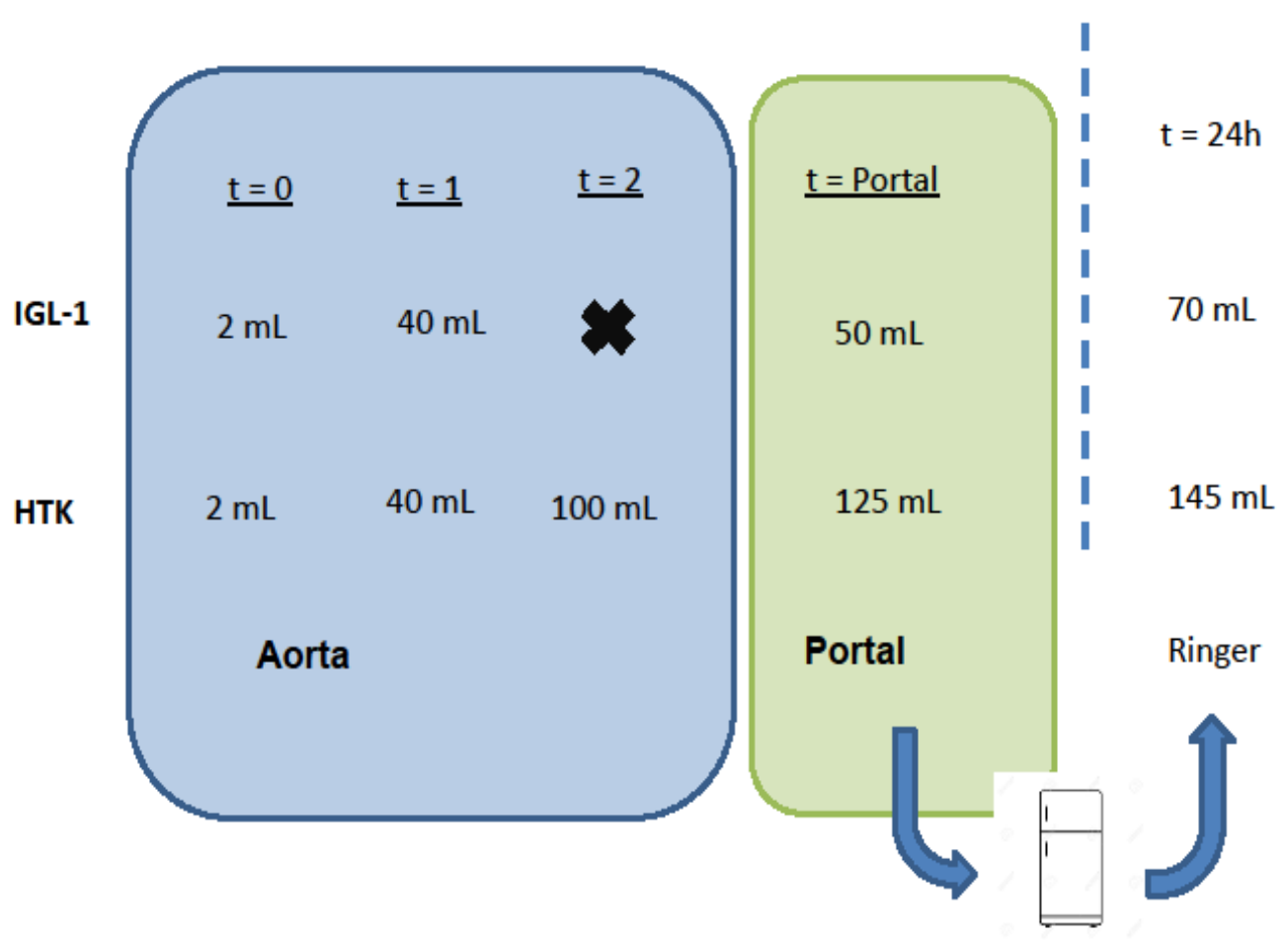


Figure 1: Washing procedures for HTK and IGL-1 solutions.

\section{A)}

RBCs

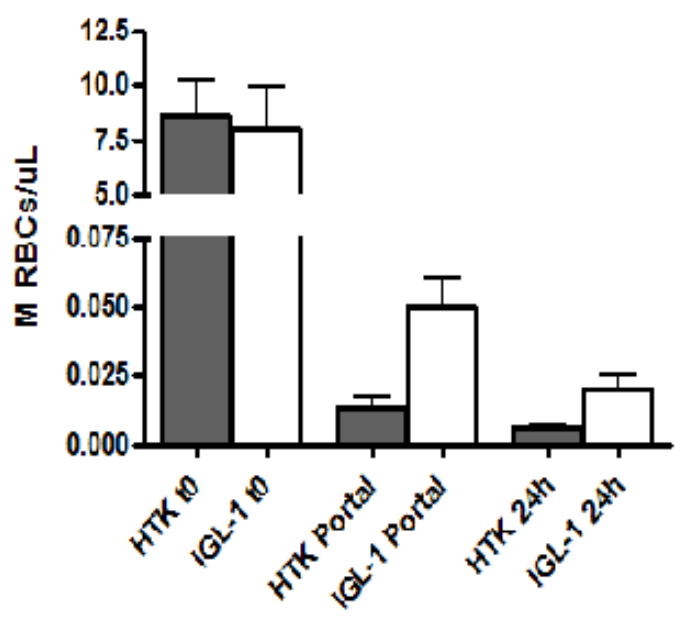

2.B)

$\mathrm{Hb}$

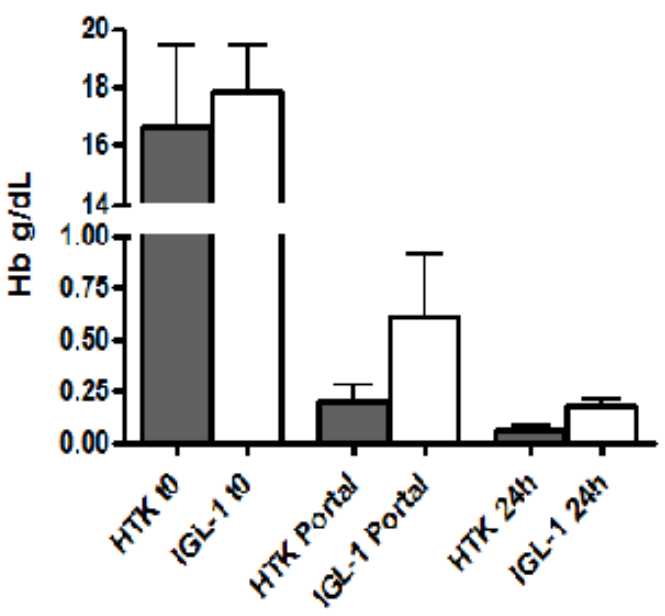

Figure 2.A: Red blood cells were counted with a Neubauer chamber and represented as cell millions/ microliter in different times for both preservation solutions according to Figure 1. Figure 2.B: Hemoglobin analyzed with Drabkins method and represented as g/dL divided in the same groups as Figure 2.A. Results are expressed as mean \pm SEM. $* p<0.05$ represent significant differences vs. HTK Portal and \# $p<0.05$ vs. HTK 24h. 
3.A)
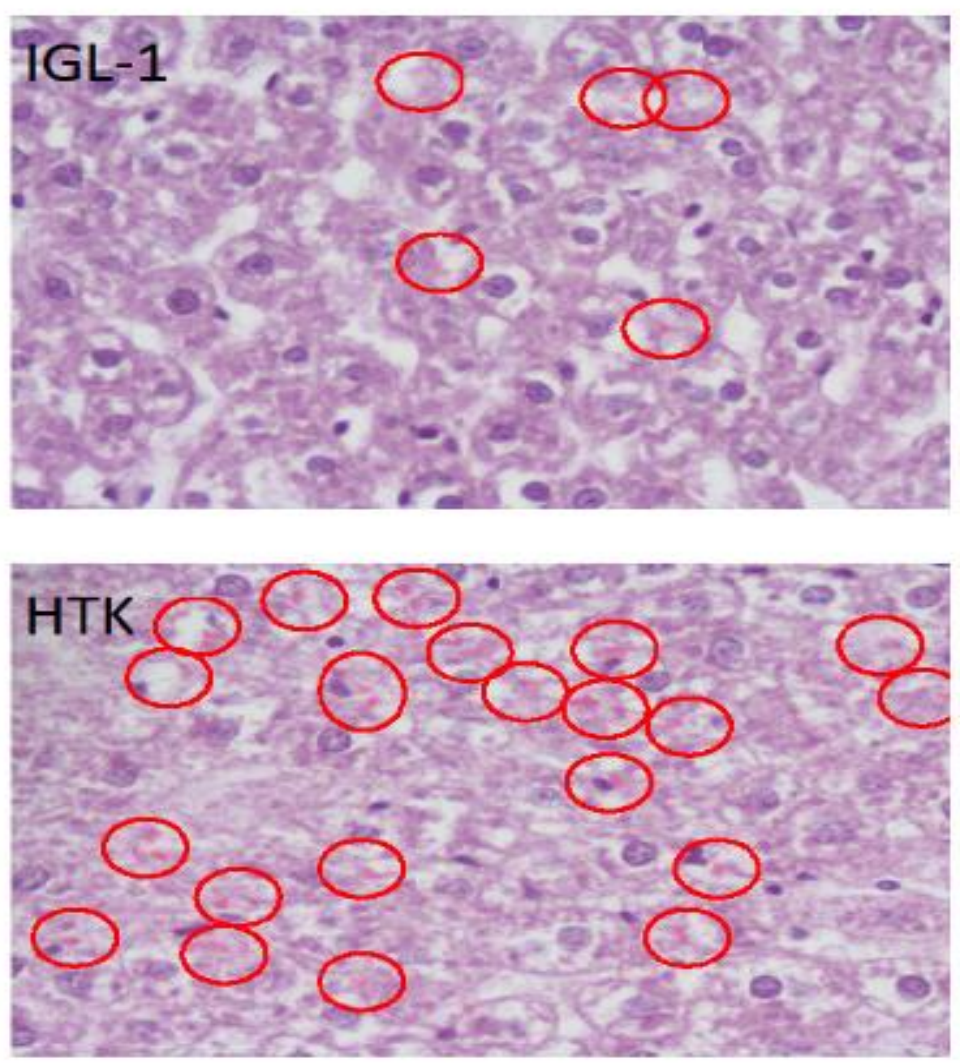

3.B)

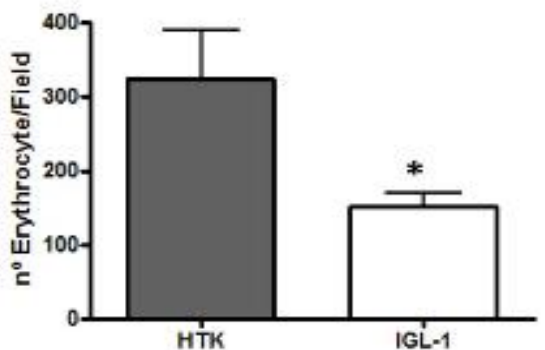


Figure 3.A: Erythrocytes were counted (red circles) after hematoxylin-eosin staining of the samples in a 40X magnification. 3.B: Erythrocites average sum from 10 random fields of each sample. Results are expressed as mean \pm SEM. $* p<0.05$ represent significant differences vs. HTK.

4.A)

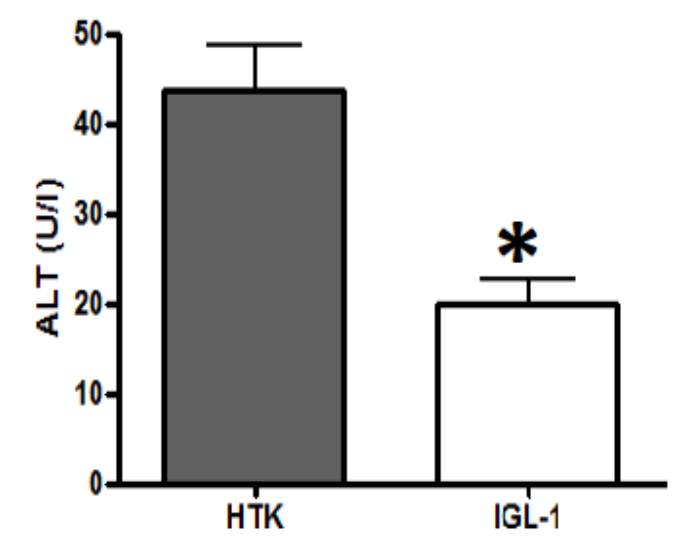

4.B)

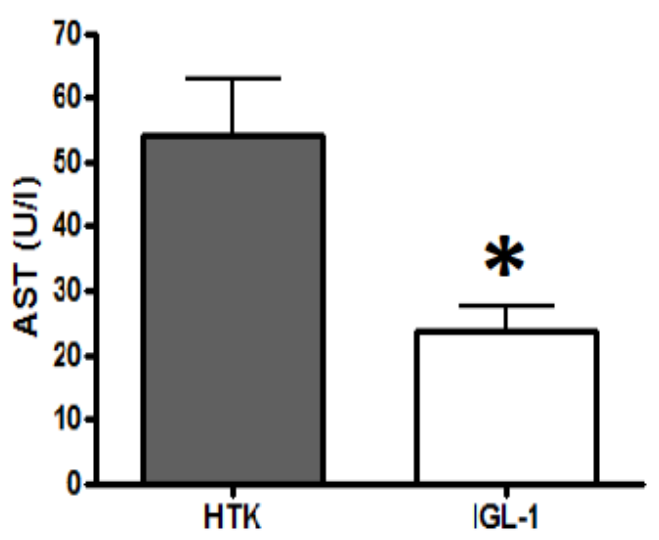

Figure 4: ALT and AST levels after 24h preservation period with HTK and IGL-1 solutions. Results are expressed as mean \pm SEM. $* p<0.05$ represent significant differences vs. HTK. 
